\title{
Harmonization vs. fragmentation: overview of climate policy scenarios in EMF27
}

\author{
Geoffrey J. Blanford • Elmar Kriegler • Massimo Tavoni
}

Received: 25 January 2013 / Accepted: 20 September 2013 / Published online: 8 January 2014

(C) Springer Science+Business Media Dordrecht 2013

\begin{abstract}
This paper synthesizes results of the multi-model Energy Modeling Forum 27 (EMF27) with a focus on climate policy scenarios. The study included two harmonized long-term climate targets of $450 \mathrm{ppm} \mathrm{CO}_{2}$-e (enforced in 2100) and $550 \mathrm{pm} \mathrm{CO}_{2}$-e (not-to-exceed) as well as two more fragmented policies based on national and regional emissions targets. Stabilizing atmospheric GHG concentrations at 450 and $550 \mathrm{ppm}$ $\mathrm{CO}_{2}$-e requires a dramatic reduction of carbon emissions compared to baseline levels. Mitigation pathways for the $450 \mathrm{CO}_{2}$-e target are largely overlapping with the $550 \mathrm{CO}_{2}$-e pathways in the first half of the century, and the lower level is achieved through rapid reductions in atmospheric concentrations in the second half of the century aided by negative anthropogenic carbon flows. A fragmented scenario designed to extrapolate current levels of ambition into the future falls short of the emissions reductions required under the harmonized targets. In a more aggressive scenario intended to capture a break from observed levels of stringency, emissions are still somewhat higher in the second half due to unabated emissions from nonparticipating countries, emphasizing that a phase-out of global emissions in the long term can only be reached with full global participation. A key finding is that a large range of energy-related $\mathrm{CO}_{2}$ emissions can be compatible with a given long-term target, depending on assumptions about carbon cycle response, non- $\mathrm{CO}_{2}$ and land use $\mathrm{CO}_{2}$ emissions abatement, partly explaining the spread in mitigation costs.
\end{abstract}

This article is part of the Special Issue on "The EMF27 Study on Global Technology and Climate Policy Strategies” edited by John Weyant, Elmar Kriegler, Geoffrey Blanford, Volker Krey, Jae Edmonds, Keywan Riahi, Richard Richels, and Massimo Tavoni.

Electronic supplementary material The online version of this article (doi:10.1007/s10584-013-0951-9) contains supplementary material, which is available to authorized users.

G. J. Blanford $(\bowtie)$

Electric Power Research Institute (EPRI), Palo Alto, CA, USA

e-mail: gblanford@gmail.com

E. Kriegler

Potsdam Institute for Climate Impact Research (PIK), Potsdam, Germany

M. Tavoni

Fondazione Eni Enrico Mattei (FEEM), Milan, Italy 


\section{Introduction}

Despite the continuing evolution of international climate policy negotiations, including the pledges made under the Copenhagen Accord in 2010 and the new pathway to a post-Kyoto agreement established by the Durban Platform in 2011, as well as a variety of other initiatives at the national and regional level, the extent and nature of mitigation effort to be undertaken by the world's economies over the long term remain highly uncertain. Many countries around the world have expressed support for a notional goal of limiting the change in global average surface temperature above the pre-industrial level to $2{ }^{\circ} \mathrm{C}$ (UNFCCC 2010), motivated by scientific findings concerning the potential impacts of warming beyond such a level (e.g. in the IPCC's Fourth Assessment Report, IPCC 2007). Due to unresolved scientific uncertainty about many complex aspects of the global climate system, it is not straightforward to map a temperature goal into a corresponding emissions pathway (Rogelj et al. 2011); nor is there consensus from a cost-benefit perspective, owing primarily to the extreme difficulty of valuing potential damages but also to more subjective questions about preferences and discounting, that such a goal is in fact optimal from a social perspective (Tol 2008).

Nonetheless, several recent studies have examined the $2{ }^{\circ} \mathrm{C}$ challenge with particular emphasis on the current context of delayed global action. See for example Jakob et al. (2012), Rogelj et al. (2012), van Vliet et al. (2012), Kriegler et al. (2013), Luderer et al. (2013), and Riahi et al. (2013). In this paper we compare and analyze results from the Energy Modeling Forum (EMF) 27 exercise, in which 18 modeling groups ran coordinated scenarios with systematic variation of both policy and technology assumptions. Our focus is the comparison of alternative policy regimes, including a fresh look at conventional long-term targets as well as two new scenarios more closely tied to potential real-world actions. The analysis explores in depth the sectoral allocation of mitigation under tight harmonized targets, which varies significantly across models, and the implications of fragmented action in the long-term. The paper first discusses methodology, then reviews results from both the global and regional perspective, and finally concludes with a summary of key findings.

\section{Methods}

The global target scenarios were specified in terms of a long-term target for radiative forcing. ${ }^{1}$ This approach has been used in the last two EMF climate scenario exercises, namely EMF21 (Weyant et al. 2006) which first introduced non- $\mathrm{CO}_{2}$ greenhouse gas mitigation into the analysis, and EMF22 (Clarke et al. 2009), which first introduced incomplete participation in addition to multi-gas analysis. In these two previous exercises, policy scenarios were constructed with a constraint on total radiative forcing from all greenhouse gases covered by the Kyoto Protocol. An important extension in EMF27 is that the constraint here is placed on a more comprehensive definition of radiative forcing, which we call AN3A forcing, inclusive of most non-gas forcing agents in addition to the forcing from greenhouse gases covered by both the Kyoto and Montreal Protocols. The Non-Kyoto substances include both warming (tropospheric ozone, black carbon,

\footnotetext{
${ }^{1}$ A target for temperature instead may prove to be a more policy-relevant formulation, but it will require further methodological work on uncertainty and the representation of climate dynamics in integrated assessment models.
} 
Montreal gases, stratospheric water vapor) and cooling substances (sulfate aerosols, organic carbon, indirect aerosol effects on cloud albedo, stratospheric ozone) ${ }^{2}$

One implication of this change is that the results across models are potentially more closely harmonized with respect to ultimate climate effects, since the more inclusive radiative forcing measure is a better (though still imperfect and uncertain) indicator of the climate response than the Kyoto subset. In addition, though non-Kyoto forcers are much less well understood and modeled than Kyoto gases, their combined forcing in 2005 was potentially large and net negative in magnitude (IPCC 2007, Chapter 2). The sign may change over time and will be model and scenario dependent, but since many of these agents are driven by fossil fuel use, its evolution will be correlated with mitigation effort. Accounting for this correlation could substantively change the dynamics of meeting the target and play an important part in determining the climate outcome of stabilization pathways (Rose et al. this issue). Two additional factors confound the inclusion of nonKyoto forcing agents in the analysis. First, most models do not have sufficient detail to describe the technologies and costs associated with reducing emissions of aerosols and ozone pre-cursors. Second, for agents thought to exhibit net negative forcing, such as sulfur aerosols, the application of a climate-focused policy would create an incentive to increase emissions, whereas local air quality and health concerns would motivate regulations to limit emissions. Resolving this trade-off is beyond the scope of this study, and beyond the capabilities of most participating models. As a consequence, models in the study were directed to control only Kyoto gas emissions, even if they include non-Kyoto substances endogenously (that is, their levels determined as functions of energy and land-use activities), but impose the nominal stabilization target on the AN3A forcing.

The AN3A forcing target levels were set to $2.8 \mathrm{~W} / \mathrm{m}^{2}$ in 2100 , allowing for overshoot before 2100 , and $3.7 \mathrm{~W} / \mathrm{m}^{2}$ that was not to be exceeded during the 21 st century. The more stringent target translates to roughly $2.4-2.7 \mathrm{~W} / \mathrm{m}^{2}$ full forcing in 2100 , and thus has a similar level of stringency as the RCP2.6 (Representative Concentration Pathway; van Vuuren et al. 2011b) that has been investigated in an ensemble of climate models (CMIP5). For simplicity, we use the labels $450 \mathrm{ppm} \mathrm{CO}_{2}-\mathrm{e}\left(=2.6 \mathrm{~W} / \mathrm{m}^{2}\right)$ and $550 \mathrm{ppm}$ $\mathrm{CO}_{2}$-e $\left(=3.7 \mathrm{~W} / \mathrm{m}^{2}\right)$ for the two targets. In both scenarios, full "where" (region), "what" (sector or source), and "when" flexibility of emissions reductions was assumed, ensuring that the least cost set of mitigation options was selected. This assumption is consistent with a globally uniform carbon price.

The study also considers two alternative policy regimes, termed the Fragmented Policy (FP) case and a scenario based on the broadly defined goal articulated in recent years by leaders in the G8. The FP and G8 scenarios differ from the stabilization scenarios in two important ways. They do not adopt a long term stabilization target, but specify emissions reductions timetables on a regional level. They also include limitations on "where" and "when" flexibility of emissions reductions, giving rise to emissions price differentials between regions and non-smooth price paths over time. The scenarios are specified based on three country groupings. Group I comprises the Annex I countries to the UNFCCC with the exception of Russia. This group acts collaboratively on climate change aiming at a joint $50 \%$ emissions reduction relative to 2005 until 2050 in the FP scenario, and an $80 \%$ reduction relative to 1990 in the G8 scenario. Group III contains

\footnotetext{
${ }^{2}$ Following the approach in van Vuuren et al. (2011b), we exclude three agents whose forcing is more speculative and often treated exogenously in the models: nitrate aerosols, mineral dust aerosols, and land use albedo changes (Abbreviated 3A). The median estimate for 3A forcing in IPCC (2007) is $-0.3 \mathrm{~W} / \mathrm{m}^{2}$. We refer to the total controlled anthropogenic forcing in this study as $\mathrm{AN}(\mathrm{ot}) 3 \mathrm{~A}$ forcing. See Rose et al. (this issue) for more details.
} 
major fossil fuel producers, in particular the Middle East OPEC countries, Russia and Former Soviet Union states of Central Asia. This group never joins the climate policy regime in either scenario (despite the reference to "all countries" in the G8 statement). Group II includes the remaining countries, including least developed countries and emerging economies such as China, India, Brazil, and South Africa (the so-called BASIC countries). They adopt their Copenhagen pledges, if available, until 2020 as domestic targets in both scenarios. After 2020 the two scenarios diverge: in FP, Group II countries adopt emissions reductions at a slowly increasing level of ambition with very limited emissions trading between them and Group I. In the G8 scenario, they join Group I countries to aim at a joint reduction of Group I+II emissions by $50 \%$ relative to 1990 in 2050, with full trade allowed between the two groups after 2020. Excerpts from the EMF27 Scenario Protocol detailing the policy specifications are provided in the Supplementary Material.

The inclusion of bottom-up policy scenarios represents another important extension in EMF 27. Rather than using models to calculate what would be required to achieve a global stabilization target (i.e. top-down framing), these scenarios begin with more tangible, potentially realistic assumptions about what commitments individual countries might make (i.e. bottom-up framing) and use models to assess both how the commitments could be met and what the implications would be for the long-term climate. Their formulation is not as clean as the global target scenarios in that a broader set of input assumptions is required, but we have attempted to define them in terms of concise storylines. The intuitive motivation for the FP case is to consider a possible future in which countries adopt targets of a stringency calibrated to a "willingness to pay" measure based on observed targets set under the Kyoto Protocol. In that instance, wealthier countries agreed to deeper cuts below a projected baseline; in the FP case in this study, (most) countries take on larger reductions as their incomes grow according to a formula derived from the observed Kyoto commitments. ${ }^{3}$ The result turns out to be a relatively weak regime in terms of the global outcome by the end of the century, although this can vary by model and across regions within a model due to variation in baseline assumptions. On the other hand, the G8 scenario is intended to capture a break from observed levels of stringency in favor of more aggressive action. Although the original articulation of the goal called for a reduction of total global emissions by $50 \%$ in 2050 (relative to 1990), we acknowledge as in the FP case that certain countries may never participate. Rather than assume compensation of non-participants by countries in the regime, we adopt an arguably more plausible interpretation in which the numerical target is applied only to the participating group of countries, which is still very inclusive. Moreover, the systematic exclusion of Group III allows an assessment of leakage and inefficiencies introduced by an incomplete coalition.

\section{Results}

Results of the model comparison across policy scenarios are divided into a discussion of global and regional outcomes. In the global context we examine the magnitude of emissions reductions across sources, climate outcomes and the role of negative emissions, and costs across scenarios with emphasis on the implications of flexibility. Secondly we explore the regional implications of policy fragmentation in terms of mitigation and costs.

\footnotetext{
${ }^{3}$ This formulation is based on the suggestion in Frankel (2007), in which negotiated reductions relative to a base year were translated into reductions from an expected baseline.
} 
3.1 Global policy outcomes: emissions and forcing

Stabilizing atmospheric GHG concentrations at 450 and $550 \mathrm{ppm} \mathrm{CO}_{2}$-e requires a dramatic reduction of $\mathrm{CO}_{2}$ emissions from fossil fuel use and industry (FF\&I) compared to baseline levels (Fig. 1a). While all models project an increase ranging from a doubling to a
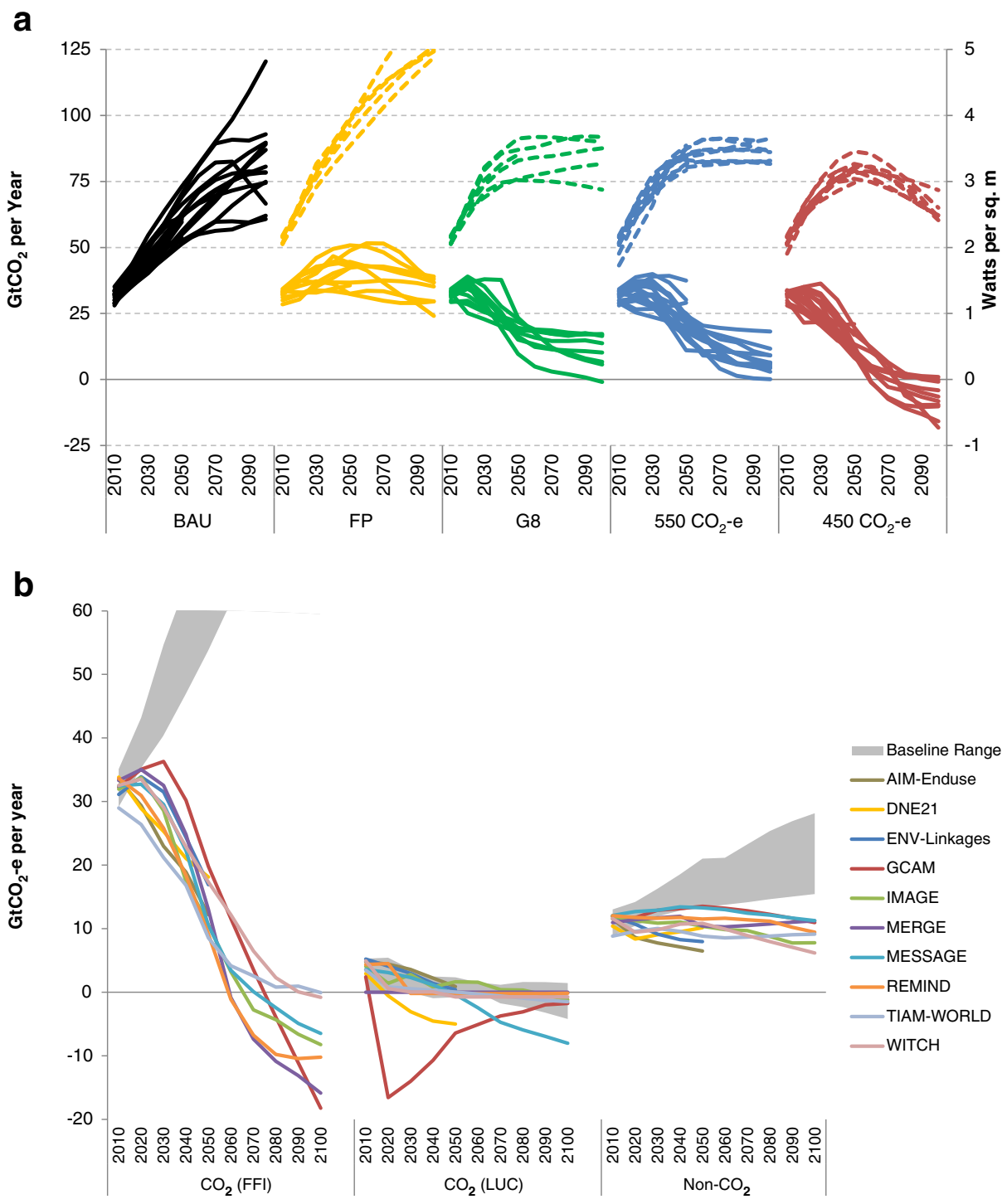

Fig. 1 Top panel (a): Global FF\&I $\mathrm{CO}_{2}$ emissions (solid lines, left axis) and Total Radiative Forcing (dashed lines, right axis) for five policy scenarios under default technology assumptions (baseline forcing not shown). Bottom panel (b): Total $\mathrm{CO}_{2}$-equivalent emissions by source (lefthand side showing $\mathrm{FF} \& \mathrm{I} \mathrm{CO}_{2}$ corresponds to top panel) for $450 \mathrm{CO}_{2}$-e case for models covering at least $\mathrm{CO}_{2}, \mathrm{CH} 4$, and $\mathrm{N} 2 \mathrm{O}$ (corresponding baseline range also shown). a NPV abatement costs (PE models) 2010-2100 (b) NPV consumption losses (GE models) 2010-2100 
quadrupling of emissions over the 21st century in the baseline, FF\& $\mathrm{I} \mathrm{CO}_{2}$ emissions are reduced by a factor of three or more from present day levels of approximately $30 \mathrm{GtCO}_{2}$ in the 550 ppm $\mathrm{CO}_{2}$-e scenario (except in model, WITCH, discussed further below), and are phased out entirely or even turn negative in the $450 \mathrm{ppm} \mathrm{CO}_{2}$-e scenario.

The increased stringency of emissions reductions in the $450 \mathrm{ppm}$ scenario is particularly evident in the 2nd half of the century. Most models show net negative $\mathrm{CO}_{2}$ emissions in the energy sector from the deployment of bioenergy with carbon capture and storage (BECCS). The negative emissions are used to compensate residual fossil fuel emissions from energy and excess emissions in the first half of the century. This solution is facilitated by the fact that the $450 \mathrm{ppm} \mathrm{CO}_{2}$-e scenario allows for overshooting of the radiative forcing target prior to 2100 . In essence, emitters borrow in the near term against the future opportunity to "print credits" via net negative emissions. The allowance for overshoot means there is no imposed limit to "indebtedness" as long as the balance is zero by the end of the century, although the deployment of negative emissions technologies in models is subject to constraints and decreasing returns to scale, as are all mitigation options. Even so, the combination of a negative emissions technology such as BECCS with a target which binds only at the end of the time horizon can lead to a particularly pronounced overshoot pattern with significant net

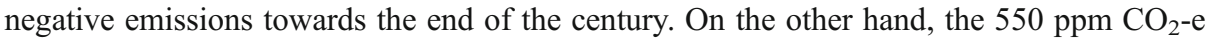
scenario not only imposes a less stringent mitigation requirement, but also does not allow for overshoot of the forcing target. The requirement that forcing never exceed the target level allows less "when" flexibility relative to the case when overshoot is allowed, since it effectively places a limit on "indebtedness" during the transition. As a consequence, the $\mathrm{CO}_{2} \mathrm{FF} \& \mathrm{I}$ emissions trajectories for the two stabilization targets turn out to be similar in the first half of the century (provided negative emissions technologies will be available).

The FP scenario, designed to extrapolate current levels of ambition into the future, falls short of the emissions reductions required under a 450 or $550 \mathrm{ppm} \mathrm{CO}_{2}$-e target. Models project $\mathrm{CO}_{2} \mathrm{FF} \& \mathrm{I}$ emissions peaking around mid-century and returning to approximately present day levels. The G8 scenario, allowing for a group of fossil resource rich countries that do not join the global regime, shows much stronger emissions reductions. Its level of stringency is in the range of the 450 and $550 \mathrm{ppm} \mathrm{CO}_{2}$-e targets for the first half of the century, but global emissions are somewhat higher in the second half due to the unabated emissions from non-participating countries, emphasizing that a phase-out of global emissions in the long term can only be reached with full global participation.

Total radiative forcing outcomes, shown in Fig. 1a and Figure S1 (supplementary material), are harmonized by design for the two global target scenarios. Some variation exists because of differences in the non-controlled agents ( $3 \mathrm{~A}$ forcing) and because of differences in calibration and timing, but for the most part the pathways are very similar across models. Forcing in the G8 case falls in a range between the 450 and $550 \mathrm{CO}_{2}$-e targets, and reaches approximately $5 \mathrm{~W} / \mathrm{m}^{2}$ (or $700 \mathrm{ppm} \mathrm{CO}_{2}$-e) in 2100 in the FP case. The extent of overshoot in the 450 $\mathrm{CO}_{2}$-e case during the century is in the range of $0.5-0.9 \mathrm{~W} / \mathrm{m}^{2}$. By contrast, as discussed in Krey et al. (this issue) and Blanford et al. (this issue), there is very little overshoot above the target when technologies associated with negative emissions are restricted. This pattern has important implications for the temperature outcome associated with the target. Because of the lag between forcing and temperature response, an overshoot forcing pathway need not lead to an overshoot temperature pathway. However, temperature is directly related to the time path of forcing, so that the greater the overshoot, the higher the temperature outcome. Although models in EMF27 report temperature change, they have varying degrees of sophistication in their climate modules, implying that findings may be less reliable than simulations with full general circulation models. These results are found in Kriegler et al. (this issue, supplementary material), 
and more generally the uncertain relationship between emissions paths and possible temperature outcomes is discussed in Rogelj et al. (2011).

Forcing and climate outcomes depend not only on the $\mathrm{CO}_{2}$ fossil fuel and industry emissions, but also on $\mathrm{CO}_{2}$ emissions from land use and emissions of non- $\mathrm{CO}_{2}$ greenhouse gases and other radiative agents. Because the non-Kyoto contribution, while potentially of large negative magnitude today, is projected to decrease significantly with mitigation (Rose et al. this issue), the imposition of a target on total forcing essentially limits the amount of cumulative Kyoto gas emissions over the 21 st century. Figure $1 \mathrm{~b}$ demonstrates the roles of the land-use and non- $\mathrm{CO}_{2}$ sources relative to the energy system in reaching the $450 \mathrm{CO}_{2}$-e goal (Figure $\mathrm{S} 2$ shows the same results for the $550 \mathrm{CO}_{2}$-e target). Non- $\mathrm{CO}_{2}$ emissions are shown in $\mathrm{CO}_{2}$-equivalent terms using 100-year GWPs.

We first note that the scale of FF\&I emissions is much greater than the other two sources, and projected baseline growth much faster, yet its emissions decline most rapidly and cumulative reductions dwarf those elsewhere. Clearly the energy sector is the primary venue for mitigation. For non- $\mathrm{CO}_{2}$ sources, which include (among others) agriculture, industry, and landfills, baseline emissions grow more slowly and (based on the current parameterization in most models) there are relatively limited opportunities for abatement. Still, the variation in emissions pathways in the policy scenario has implications for allowable emissions from other sources. For example, the WITCH model has the lowest non- $\mathrm{CO}_{2}$ trajectory and the highest FF\&I trajectory (also for the $550 \mathrm{CO}_{2}$-e case, as observed above). Finally, the role of land-use emissions is potentially important, but only a few models fully incorporate land-based activities into the mitigation strategy. Most notable is the GCAM model, in which an extremely rapid decrease in LUC emissions (i.e. net increase in the terrestrial $\mathrm{CO}_{2}$ sink through afforestation) allows a delayed decline in emissions in the energy sector. Towards the end of the century however, GCAM projects a decline in this negative sink as land use for biomass production increases to facilitate negative emissions in the energy sector through BECCS. By contrast, the MESSAGE model shows the opposite trend with an increasingly negative flow in both LUC and FF\&I emissions.

Further, it is observed that models show a large spread in cumulative (2011-2100) total Kyoto gas emissions: $550 \mathrm{GtCO}_{2}$-e (1750-2300 $\mathrm{GtCO}_{2}$-e) and $800 \mathrm{GtCO}_{2}$-e (2460-3260 $\mathrm{GtCO}_{2}$-e) for the 450 and $550 \mathrm{ppm} \mathrm{CO}_{2}$-e stabilization targets, respectively (Kriegler et al. this issue, supplementary material). A key factor for this spread is variation in the treatment of the carbon cycle, in particular the extent of temperature feedbacks and other changes in uptake that impact the evolution of the airborne fraction, that is, the portion of emitted carbon retained in the atmosphere (Figures S3 and S4 in Supplementary Material). Thus uncertainty about the carbon cycle has a considerable influence on the remaining $\mathrm{CO}_{2}$ budget under a stabilization target. It is particularly relevant for low stabilization targets where the range of variation is large relative to the allowable budget and therefore has a greater impact on mitigation pathways and costs. We also note that the dynamics of the airborne fraction of anthropogenic $\mathrm{CO}_{2}$ are important for the ability to temporarily overshoot the forcing target with the use of negative emissions. The slower the airborne fraction declines due to climate feedbacks, the more limited the ability to overshoot. An appropriate treatment of the carbon cycle and the associated uncertainty is therefore important for the assessment of low stabilization pathways. ${ }^{4}$

Model differences in sectoral allocation of effort are compounded by the variation in carbon cycle formulation, resulting in even larger spread for the admissible $\mathrm{CO}_{2}$ budget from fossil fuel

\footnotetext{
${ }^{4}$ Analysis of the variation of carbon cycle modules in integrated assessment models and how they compare to more complex carbon-cycle models has been conducted by Hof et al. (2012) van Vuuren et al. (2011a), and Arora et al. (2013).
} 
use and industry. The cumulative FF\& $\mathrm{I} \mathrm{CO}_{2}$ emissions from 2011 through 2100 (i.e. the integral of the time paths shown in the figure) vary by a factor of two between the models (680-1400 $\mathrm{GtCO}_{2}$ in the 450 ppm $\mathrm{CO}_{2}$-e case and $1260-2340 \mathrm{GtCO}_{2}$ in the $550 \mathrm{ppm} \mathrm{CO}_{2}$-e case; Kriegler et al. this issue, supplementary material). These observations culminate in a fundamentally important point: with so many systems and sources acting on the final outcome expressed in terms of a target forcing level, and with considerable uncertainty about how those systems work or may evolve in the future, many different combinations of mitigation activities can be modeled as consistent with the same outcome. Thus, while the results of this study do provide some compelling insights into the scale of mitigation involved and the roles of key technologies and sources, they do not support specific conclusions about what is "required" in any particular sector to meet ambitious climate goals. Rather the breadth of participating models demonstrates a variety of alternative pathways and strategies.

\subsection{Global policy outcomes: mitigation costs}

Global policy cost metrics include both a price on carbon, reflecting the stringency of the mitigation requirement at the margin, and a gross economic cost. For comparability across models, we adopt an economic cost metric equal to consumption loss relative to baseline when available, substituted by proxy measures in partial equilibrium models, expressed as a fraction of baseline GDP. Note that this metric does not incorporate potential climate damages; we have undertaken a cost-effectiveness analysis only for the targets specified. For simplicity, we present here an average of carbon prices over the 2010-2100 horizon weighted by a discount factor (but not by emissions) and a net present value (NPV) total for economic costs. ${ }^{5}$ Although there are important dynamic effects in certain cases, collapsing the time dimension preserves the essential insights.

For the global stabilization regimes at 450 and $550 \mathrm{ppm} \mathrm{CO}_{2}$-e, the study protocol stipulated universal emissions pricing across regions and sources to ensure the selection of the cheapest globally available mitigation option at the margin. The reported time-average carbon price for the $550 \mathrm{CO}_{2}$-e target ranged from $\$ 5$ to $\$ 50$ per $\mathrm{tCO}_{2}$ and for the $450 \mathrm{CO}_{2}$-e target from $\$ 12$ to $\$ 92$ per $\mathrm{tCO}_{2}$ (Figure S5, supplementary material). ${ }^{6}$ Reported NPV total economic costs reflect a similarly broad range, from a $0.4 \%$ to $8.0 \%$ relative to baseline for the $550 \mathrm{CO}_{2}$-e target and from $0.8 \%$ to $11.7 \%$ for the $450 \mathrm{CO}_{2}$-e target (Fig. 2-note different metrics described in caption). Despite the wide range of variation, both the carbon price and the economic cost metrics increase by roughly a factor of 1.5 to 2.5 from the 550 to the $450 \mathrm{CO}_{2}$-e target (with a few exceptions).

The range of costs emerges for a variety of reasons. First, the scale of the mitigation requirement to meet a physical target depends on model baseline assumptions as well as on the representation of physical systems, as discussed above. Moreover, the varying extent of mitigation options in land-use and non- $\mathrm{CO}_{2}$ emissions implies a broader range for the scale of effort within the energy sector, which has the dominant share of abatement and also in most cases the marginal abatement activity. ${ }^{7}$ To control for these effects, Fig. 2 and Figure S5 plot the cost metrics against mitigation scale as measured by the fraction of cumulative $\mathrm{FF} \& \mathrm{I} \mathrm{CO}_{2}$ emissions abated. Comparing the $450 \mathrm{CO}_{2}$-e results to those for the $550 \mathrm{CO}_{2}$-e scenario, the

\footnotetext{
${ }^{5}$ In both cases a discount rate of $5 \%$ was used ex post, though it should be noted that individual models may use different rates and treat time preferences differently.

${ }^{6}$ Note that in all cases the price rises significantly over time, so that high future levels are suppressed by reporting only the time-average price.

${ }^{7}$ Models generally assume that the land use and non- $\mathrm{CO}_{2}$ sources are characterized by a limited amount of low cost mitigation options followed by steeply rising marginal costs of further emissions reductions (Figure S6). Once these options are exhausted, additional reductions must occur in the energy sector, whose marginal abatement cost curve is less convex. Further research on non-energy abatement options could change this characterization.
} 
a

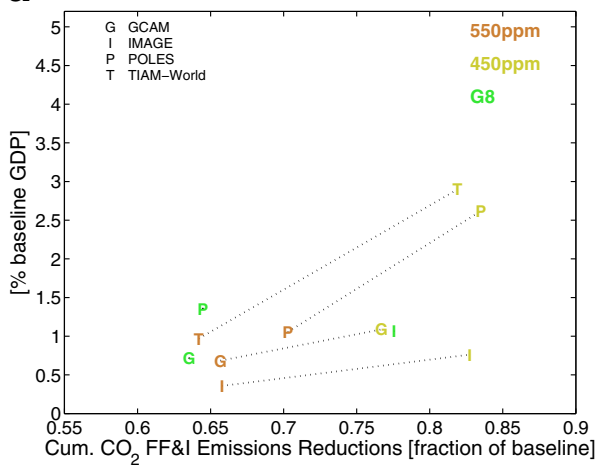

b

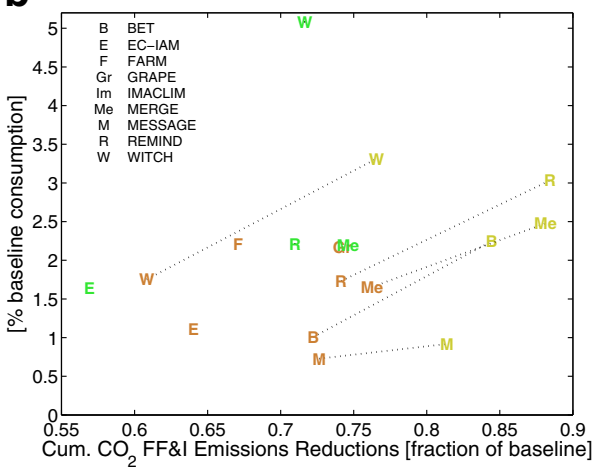

Fig. 2 Global net present value (NPV) policy costs for the period 2010-2100 (discounted at $5 \%$ ) for the 450 and $550 \mathrm{ppm} \mathrm{CO} 2 \mathrm{e}$ targets and the G8 policy scenario. Includes only models with results reported to 2100 . The partial equilibrium (PE) models report costs in terms of area under the marginal abatement cost curve (expressed in percentage of GDP in the baseline); the general equilibrium (GE) models report consumption losses (expressed in percentage of consumption in the baseline). Costs are plotted against cumulative $\mathrm{FF} \& \mathrm{I} \mathrm{CO}_{2}$ emissions reductions as a fraction of cumulative emissions in the baseline. Dashed lines connect 450 and $550 \mathrm{CO}_{2}$-e scenario results for individual models producing both cases. Outside the plotted range are consumption losses for IMACLIM at $8.0 \%$ (550 ppm), $11.7 \%$ (450 ppm) and $6.7 \%$ (G8) for the period 2010-2100

majority of models shows similar cost increases in the range of 1-1.5\% NPV losses for an additional $15 \%$ reduction of cumulative FF\&I baseline emissions above $550 \mathrm{ppm} \mathrm{CO}_{2}$-e levels, with a few models showing lower cost increases from a lower cost base. Beyond scale, other important differences include the cost and availability of mitigation technologies and options, including substitution opportunities on the demand side, and certain aspects of model structure. The option for negative emissions, e.g. through BECCS, can play a strong role in determining costs and feasibility particularly for the $450 \mathrm{CO}_{2}$-e target. ${ }^{8}$ It is important to note that a larger set of models have calculated the $550 \mathrm{ppm} \mathrm{CO}_{2}$-e than the $450 \mathrm{ppm} \mathrm{CO}_{2}$-e target so that a comparison of cost ranges between the two is subject to a sampling bias (Tavoni and Tol 2010). A special case is the IMACLIM model, which includes a more extensive treatment of non-carbon distortions and externalities. In this setting the introduction of a carbon price without accompanying measures to address other market failures leads to much higher economic costs (Bibas and Méjean 2013).

Figure 2 also demonstrates that the G8 scenario results in less mitigation at a higher cost relative to the "frontier" implied by the line linking the two global target scenarios with full flexibility. The inefficiency results from forgoing available abatement options in the non-participatory Group III countries, and also from the limited "when" flexibility implied by the requirement to meet a specific emissions goal in 2050. Not shown are the costs for the FP scenario, which is much less stringent than the other three but more inefficient due to the enforcement of specific emissions targets on individual countries and regions with limits to trading (which also leads to different carbon prices in different regions). For very stringent mitigation goals, these sources of inefficiency could significantly increase the costs of an already ambitious endeavor.

\subsection{Implications of regional fragmentation}

We conclude our results by assessing the impacts of the four climate policies analyzed in this study on developed (Group I), currently developing (Group II) and energy-exporting regions

\footnotetext{
${ }^{8}$ See Krey et al. (this issue) for further discussion on the impact of technology availability on costs.
} 

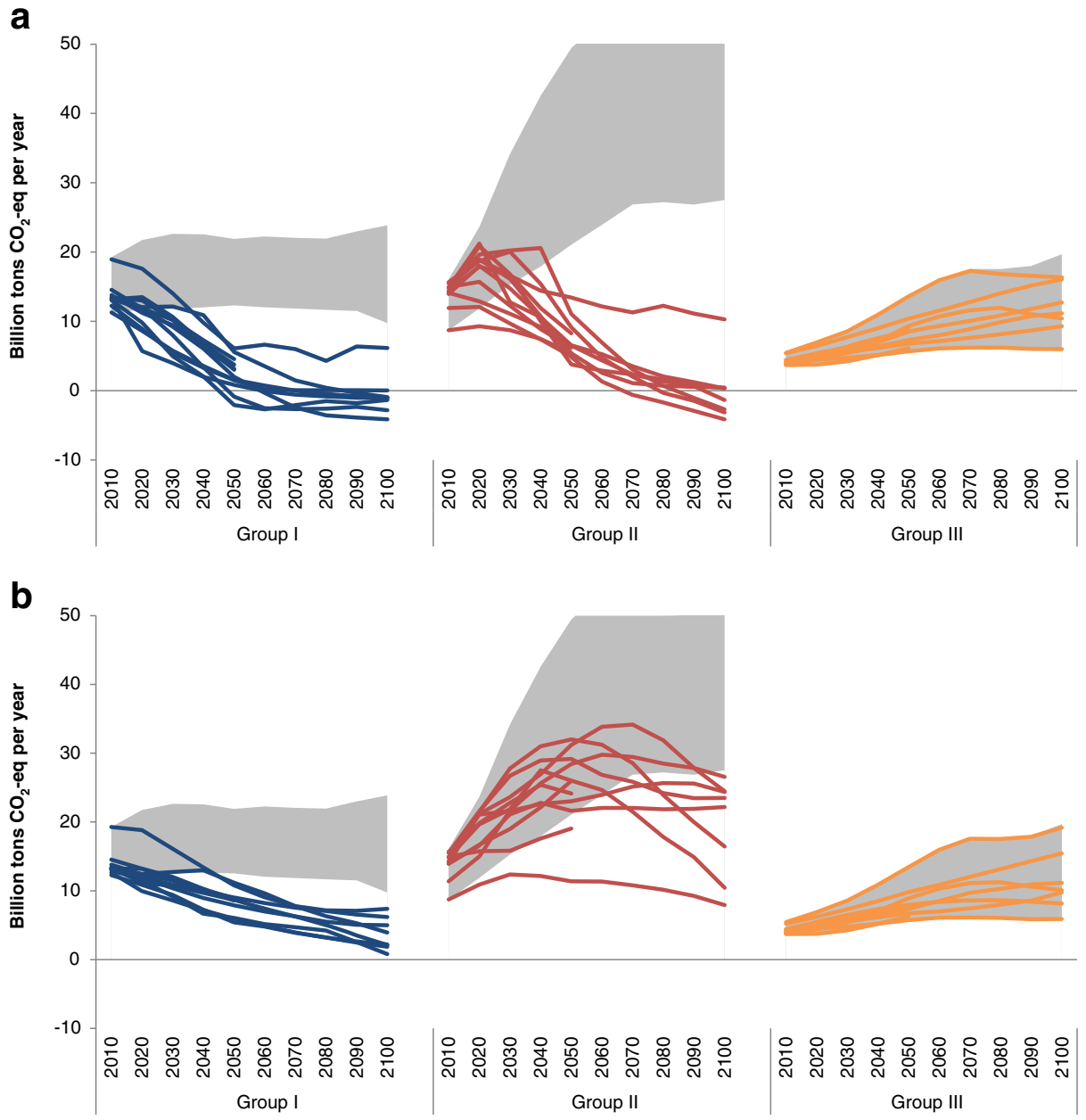

Fig. 3 Top panel (a): G8 Scenario FF\&I CO 2 Emissions by Group; Bottom panel (b): FP Scenario FF\&I CO 2 Emissions by Group. Includes all models reporting scenario results. Regional definitions vary by model, accounting for some of the base year discrepancy. Corresponding baseline ranges shown in grey

(Group III). Figure 3 shows FF\&I $\mathrm{CO}_{2}$ emissions in the three groups in the FP and G8 policy scenarios relative to the baseline. It is clear that future growth in the baseline is driven almost entirely by Group II and III countries (see Blanford et al. 2012). Emissions from non-participating Group III become large relative to residual emissions in the rest of the world, especially in the G8 case. The major difference between the two policies is the extent of mitigation in Group II countries, which take on an extremely aggressive commitment in the G8 case and a more modest (but not trivial) one in the FP scenario, although the implications of the income-based reduction formula vary widely by model. ${ }^{9}$

\footnotetext{
${ }^{9}$ One consequence of the bottom-up formulation is that model differences in relative growth rates across regions are emphasized in these scenarios, revealing an important dimension of variation and uncertainty that tends not to emerge as clearly with a harmonized target.
} 
One of the most policy relevant implications of integrated assessment modeling of climate mitigation pathways is the extent to which countries and regions are affected by alternative policy regimes. Since participation in any international agreement is voluntary, particular scrutiny will be given domestically to the technical, economic, and social repercussions of policies before joining a coalition, especially relative to the other members. Figure 4 shows the distribution of costs and abatement across the three groups for the 550 ppm-eq policy (results are similar for the 450 case). In this study, the target has been implemented with an efficient allocation of abatement across regions-no redistribution through $\mathrm{CO}_{2}$ permit allocation was indicated (though individual models may use different assumptions). It thus represents a limiting case in which all countries face an equal carbon price without any ex post compensation (an unlikely political outcome but an important starting point for analysis). Models find that in this setting, policy costs are significantly greater in developing and energy exporting regions than in industrialized countries (as in Tavoni et al. 2013). The diversity of policy costs across regions is a striking result, though it is not a new one in the literature (e.g. Clarke et al. 2009). Given that all regions face the same price of carbon, it is intuitive to attribute the disparity in costs to a different level of abatement: if developing countries have more abatement opportunities (e.g. due to more inefficient capital), they will abate more and incur larger economic losses. However, the right panel of Fig. 4 shows that, cumulatively to 2050, abatement compared to industrialized countries is proportionally similar for energy-exporters, and only somewhat higher in developing countries.

This finding suggests that there are important additional drivers of regional differences in policy costs (see also Tavoni et al. 2013). We highlight two examples. First, developing countries devote a greater fraction of their economy to energy; this is particularly important when regional economies are aggregated using market exchange rates, the current practice for most participating models. Second, terms of trade related to the global markets (especially, but not exclusively, energy markets) are very important for energyexporting countries. For example, climate policies would simultaneously reduce the consumption and price of fossil fuel products, with important repercussions on economies highly dependent on energy revenues. Indeed, Group III (orange markers) is the region most penalized in terms of (uncompensated) policy costs. Given the strategic role which this
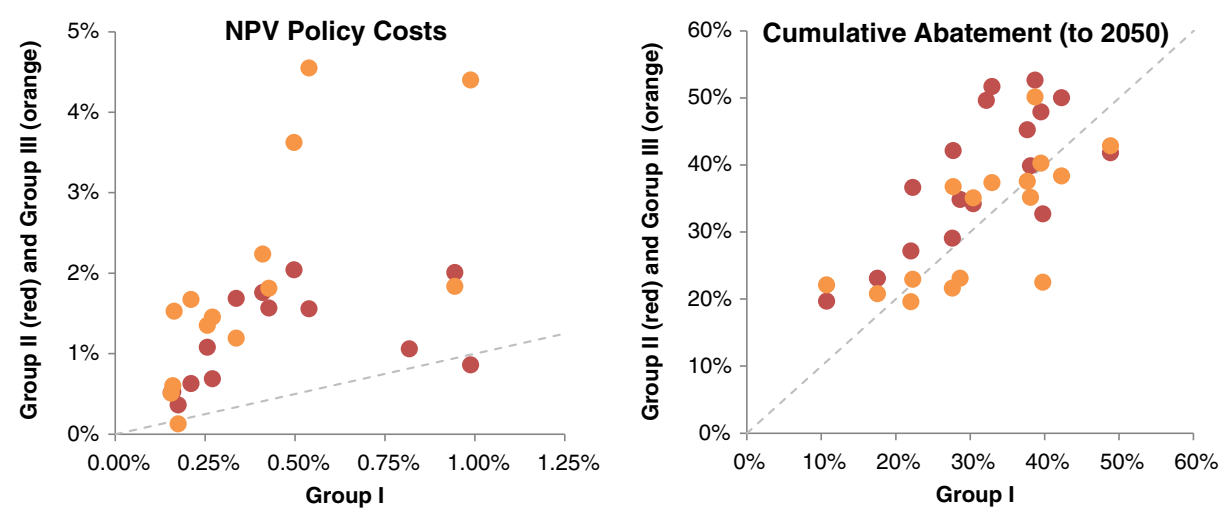

Fig. 4 Distribution across groups of NPV policy costs (left panel) and cumulative abatement (right panel) for the $550 \mathrm{ppm} \mathrm{CO}_{2}$-e policy. Group I is on the X-axis, and Groups II and III are on the y-axis, identified by red and orange markers respectively. The dashed line indicates where the effort (in terms of costs and mitigation) is equal across groups 


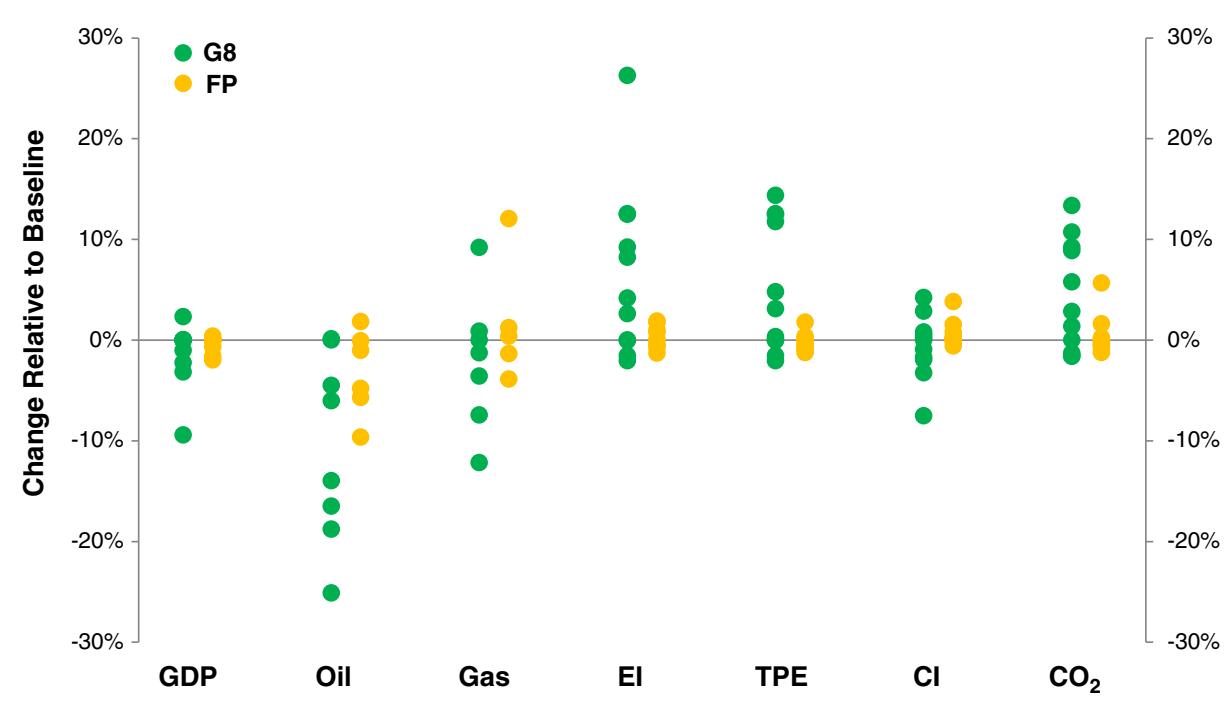

Fig. 5 Change in Group III Indicators in 2050 Relative to Baseline in G8 and FP Scenarios. Individual model results plotted for GDP, cumulative oil and gas extraction, energy intensity of GDP, total primary energy (TPE), carbon intensity (CI), and energy-related $\mathrm{CO}_{2}$ emissions

part of the world plays in international affairs, more analysis is warranted (see Leimbach et al. (2009) and Massetti and Tavoni (2011)).

The G8 and FP scenarios excluded explicit mitigation commitment in Group III out of the recognition that involving energy exporting countries in international climate agreements is a challenging endeavor. General equilibrium effects, e.g. through changes in global market prices, would nonetheless impact the region even in the absence of a domestic climate policy. A decomposition of the major effects is shown in Fig. 5. Economic activity, as measured by GDP, contracts in most models in both the FP and G8 policies, and especially in the latter, despite the lack of a mitigation requirement. This result is not trivial since the terms of trade effect on energy markets could in principle be compensated by relocation of energy intensive industries. ${ }^{10}$ Second, oil extraction-which is the dominant source of revenues in energy markets - also contracts, with a reduction of up to $25 \%$ in cumulative extraction through 2050. Extraction is also not done in advance, providing no indication of a 'green paradox' effect in which oil suppliers increase quantity in response to a carbon price (Sinn 2012). Some models exhibit a partial compensation by increased extraction of gas (a less carbon intensive alternative), while others depict contraction in both markets due to a shift to low carbon sources and lower energy demand. Third, total primary energy increases in most models both in total and relative to GDP (energy intensity), suggesting a 'rebound effect' due to lower energy prices. In half of the models carbon intensity declines, as the reduced demand for gas and oil by Groups I and II forestalls their replacement by more carbon intensive fuels in Group III; others project increases in carbon intensity. The resulting impact on emissions indicates moderate leakage, stronger in the G8 scenario, with a maximum of $13 \%$, while a few models show emissions reductions driven by reductions in carbon intensity.

\footnotetext{
${ }^{10}$ Only a few models represent energy-intensive industries and trade in non-energy goods with sufficient disaggregation to directly assess the potential extent of "off-shoring".
} 


\section{Conclusions}

In the last EMF study of global climate policies (EMF22, Clarke et al. 2009), roughly half of participating models found a feasible solution for the $450 \mathrm{ppm} \mathrm{CO}_{2}$-e target, even allowing for overshoot. In this study, nearly every model can find a feasible pathway. Two factors explain this change: first, by including non-Kyoto forcing agents in the definition of $\mathrm{CO}_{2}$ equivalent forcing, the current median estimate of total forcing is further below the target level (although this is less relevant in the context of an overshoot target). Second and more importantly, more models have added a technological option for negative emissions, in most cases BECCS in the electric sector. With this option, the mitigation pathways identified by models for the $450 \mathrm{CO}_{2}$-e target are largely overlapping with the $550 \mathrm{CO}_{2}$-e not-to-exceed pathways in the first half of the century, and the lower level is achieved through rapid reductions in atmospheric concentrations in the second half of the century aided by negative anthropogenic carbon flows. This pattern notwithstanding, participating models exhibit diverse combinations of mitigation across sources, depth of overall cuts, and gross and marginal costs for the same target. A key finding is that a large range of cumulative $\mathrm{CO}_{2}$ emissions from fossil fuel use and industry can be compatible with the 450 and $550 \mathrm{ppm}$ $\mathrm{CO}_{2}$-e stabilization targets, depending on assumptions about carbon cycle response, Non$\mathrm{CO}_{2}$ and land use $\mathrm{CO}_{2}$ emissions abatement. Part of the spread in mitigation costs can be explained by the differences in the scale of the FF\&I CO $\mathrm{CO}_{2}$ emissions reductions.

This study introduces two policy scenarios formulated in terms of national and regional emissions constraints to counter the globally harmonized targets. A stylized implementation of the G8 target including the assumption of a group of non-participating countries results in limiting forcing to the range between 450 and $550 \mathrm{CO}_{2}$-e, but with higher costs relative to the cumulative level of abatement than the globally harmonized pathway. The FP scenario representing an extrapolation of demonstrated levels of ambition results in forcing around $700 \mathrm{CO}_{2}$-e by the end of the century, in most cases still growing. Models depict higher economic costs in developing countries for comparable or slightly higher levels of abatement in a setting with no ex post compensation, and energy-exporting countries could incur costs even in a regime from which they abstain. The results emphasize the importance of international cooperation and a break from the current trajectory of policy commitments if climate outcomes in the vicinity of the $2{ }^{\circ} \mathrm{C}$ goal are to be achieved. The dependence of this goal on negative emissions in future decades must be brought to the fore in the policy debate.

Acknowledgments The authors are grateful to three anonymous reviewers for their insightful comments. The contributions of Elmar Kriegler and Massimo Tavoni to this research was supported by funding from the European Union's Seventh Framework Programme FP7/2011 under grant agreement no. 282846 (LIMITS).

\section{References}

Arora VK et al (2013) Carbon-concentration and carbon-climate feedbacks in CMIP5 earth system models. J Clim 26:5289-5314

Bibas R, Méjean A (2013) Potential and limitations of bioenergy for low carbon transitions. Climatic Change. doi:10.1007/s10584-013-0962-6

Blanford GJ, Rose SK, Tavoni M (2012) Baseline projections of energy emissions in Asia. Enegry Econ 34:S284-S292

Blanford GJ et al (this issue) Trade-offs between mitigation costs and temperature change. Clim Chang, this issue. doi:10.1007/s10584-013-0869-2 
Clarke LE et al (2009) International climate policy architectures: overview of the EMF22 international scenarios. Energy Econ 31:S64-S81

Frankel J (2007) Formulas for Quantitative Emissions Targets. In: Aldy JE, Stavins RN (eds), Architectures for Agreement. Cambridge University Press, pp 31-56

Hof AF et al (2012) The benefits of climate change mitigation in integrated assessment models: the role of the carbon cycle and climate component. Clim Chang 113:897-917

IPCC (2007) Climate Change 2007: The Physical Science Basis. In: Solomon S et al (eds), Contribution of Working Group I to the Fourth Assessment Report of the Intergovernmental Panel on Climate Change. Cambridge University Press

Jakob M et al (2012) Time to act now? Assessing the costs of delaying climate measures and benefits of early action. Clim Chang 114:79-99

Krey V et al (this issue) Getting from here to there - energy technology pathways in the EMF27 scenarios. Clim Chang, this issue. doi:10.1007/s10584-013-0947-5

Kriegler E et al (2013) Can we still meet $2{ }^{\circ} \mathrm{C}$ with global climate action? The LIMITS study on implications of Durban Action Platform scenarios. Clim Chang Econ, in revision

Kriegler E et al (this issue) The role of technology for climate stabilization: overview of EMF27 study on energy system transition pathways under alternative climate policy regimes. Clim Chang, this issue. doi:10.1007/s10584-013-0953-7

Leimbach $\mathrm{M}$ et al (2009) Mitigation costs in a globalized world: climate policy analysis with REMIND-R. Environ Model Assess 15(3):155-173

Luderer $\mathrm{G}$ et al (2013) Implications of weak near-term climate policies on long-term mitigation pathways. Clim Chang. doi:10.1007/s10584-013-0899-9

Massetti E, Tavoni M (2011) The cost of climate change mitigation policy in Eastern Europe and Former Soviet Union. Clim Chang Econ 2(4):341-370

Riahi K et al (2013) Locked into Copenhagen Pledges-Implications of short-term emission targets for the cost and feasibility of long-term goals. Technol Forecast Soc Chang, in revision

Rogelj J et al (2011) Emissions pathways consistent with a $2{ }^{\circ} \mathrm{C}$ global temperature limit. Nat Clim Chang 1:413-441

Rogelj J et al (2012) 2020 emissions levels required to limit warming to below $2{ }^{\circ} \mathrm{C}$. Nat Clim Chang. doi: $10.1038 /$ nclimate 1758

Rose SK et al (this issue) The role of non-Kyoto gas forcing in GHG stabilization. Clim Chang, this issue. doi:10.1007/s10584-013-0955-5

Sinn H-W (2012) The Green Paradox: A Supply-Side Approach to Global Warming. MIT Press

Tavoni M, Tol RSJ (2010) Counting only the hits? The risk of underestimating the costs of stringent climate policy. Clim Chang 100:769-778

Tavoni M et al (2013) The distribution of the major economies' effort in the Durban platform scenarions. Clim Chang Econ (accepted)

Tol RSJ (2008) The social cost of carbon: trends, outliers and catastrophes. Econ Open Access Open Assess E Jl 2:2008-25

UNFCCC (2010) Report of the COP16, Cancun, December 2010, Addendum: Decision 1/CP16, found at: http://unfccc.int/resource/docs/2010/cop16/eng/07a01.pdf

van Vliet et al (2012) Copenhagen accord pledges imply higher costs for staying below $2^{\circ} \mathrm{C}$ warming. Clim Chang 113:551-561

van Vuuren et al (2011a) How well do integrated assessment models simulate climate change? Clim Chang 104(2):255-285

van Vuuren et al (2011b) The representative concentration pathways: an overview. Clim Chang 109(1-2):5-31

Weyant JP, de la Chesnaye FC, Blanford GJ (2006) Overview of EMF 21: Multigas Mitigation and Climate Policy. Energy J, Multi-Greenhouse Gas Mitiation and Climate Policy Special Issue, pp 1-32 